\title{
IUFOST2006/1089 \\ Sensory Science and Consumer Behavior: Current Practices and Applications
}

\author{
H. Stone and J. Sidel
}

Tragon Corporation, 365 Convention Way, Redwood City, CA 94063, United States of America hstone@tragon.com

\begin{abstract}
Measuring consumer attitudes and perceptions are a critical part of a product's success in the marketplace. Both sensory and attitudinal measures are being used with increasing frequency but often with a lack of appreciation for the complexities of the human perceptual system. This presentation will explore current practices for selecting consumers for sensory tasks vs those selected for attitudinal tasks. While most all consumers are able to express a preference, a smaller percentage can describe perceptions with any degree of sensitivity and reliability. These aspects of consumer behavior must be addressed in advance of any test if the results are to be believed and used effectively. Recommended practices will be reviewed along with examples of applications. New developments in measuring consumer attitudes also will be described along with their applications.
\end{abstract}

